Necessary and sufficient condition on the fixed-energy scattering data for the potential to be spherically symmetric

This article has been downloaded from IOPscience. Please scroll down to see the full text article.

1989 Inverse Problems 5 L45

(http://iopscience.iop.org/0266-5611/5/5/001)

View the table of contents for this issue, or go to the journal homepage for more

Download details:

IP Address: 194.95.184.111

The article was downloaded on 29/06/2011 at 08:24

Please note that terms and conditions apply. 


\title{
Necessary and sufficient condition on the fixed-energy scattering data for the potential to be spherically symmetric
}

\author{
A G Ramm $\dagger$ and O L Weaver $\ddagger$ \\ $\dagger$ Mathematics Department, Kansas State University, Manhattan, KS 66506, USA \\ $\ddagger$ Strahlenzentrum, University of Giessen, 6300 Giessen, Federal Republic of Germany, \\ and Physics Department, Kansas State University, Manhattan, KS 66506, USA
}

Received 20 June 1989

\begin{abstract}
Consider the Schrödinger equation for fixed energy and a real-valued compactly supported potential $q(x) \in L_{\mathrm{loc}}^{2}, x \in \mathbb{R}^{3}$. Let $A\left(\theta^{\prime}, \theta\right)$ be the corresponding scattering amplitude for incident and exit directions $\theta$ and $\theta^{\prime}$ respectively. It is proved that the potential is spherically symmetric if and only if $A\left(\theta^{\prime}, \theta\right)$ for this single energy depends only on the angle between $\theta$ and $\theta^{\prime}$, that is $A\left(\theta^{\prime}, \theta\right)=A\left(\theta^{\prime} \cdot \theta\right)$ for all $\theta^{\prime}, \theta \in S^{2}$, the unit sphere. Thus, if this condition holds at a single energy $\kappa^{2}>0$ it holds for all $\kappa>0$ under the above assumption about $q(x)$.
\end{abstract}

Let

$$
\left[\nabla^{2}+\kappa^{2}-q(x)\right] u=0 \quad \text { in } \mathbb{R}^{3} \quad \kappa>0 \text { is fixed. }
$$

Without loss of generality let us assume in what follows that $\kappa=1$. The scattering solution is defined as the solution to (1) with the asymptotics

$u=\exp (\mathrm{i} \theta \cdot x)+A\left(\theta^{\prime}, \theta\right) r^{-1} \exp (\mathrm{i} r)+o\left(r^{-1}\right) \quad$ as $r=|x| \rightarrow \infty, x r^{-1}=\theta^{\prime}$.

The function $A\left(\theta^{\prime}, \theta\right)$ is the scattering amplitude. Let us assume

$$
q \in Q_{a}:=\left\{q: q(x)=\bar{q}(x) \in L^{2}\left(B_{a}\right), q(x)=0 \text { in } B_{a}^{\prime}\right\}
$$

where $B_{a}=\{x:|x| \leqslant a\}, B_{a}^{\prime}:=\mathbb{R}^{3} \backslash B_{a}$. If $q(x)=q(r), r=|x|$, then it is well known that

$$
A\left(\theta^{\prime}, \theta\right)=A\left(\theta^{\prime} \cdot \theta\right) \quad \forall \theta^{\prime}, \theta \in S^{2}
$$

where $S^{2}$ is the unit sphere in $\mathbb{R}^{3}$. The basic result of this letter, theorem 1 , has been formulated as a conjecture in [1].

Theorem 1. Assume that (3) holds. Then $q(x)=q(r)$ iff (4) holds. Thus (4) holds at all energies iff it holds at one.

The result of this type in the case when $A\left(\theta^{\prime}, \theta, \kappa\right)=A\left(\theta^{\prime} \cdot \theta, \kappa\right)$ for all $\theta^{\prime}, \theta \in S^{2}$ and all $\kappa>0$ has been proved in [1]. In [2-4] a theory for inverse scattering problems with fixed-energy data is given and in [5-6] one finds basic properties of the scattering amplitude. Our proof is very short and is based on the uniqueness theorem proved in $[2-4,7,8]$. 
Proof. First we formulate the uniqueness theorem from [4].

Theorem 2. If $q_{j} \in Q_{a}, j=1,2$, and $A_{1}\left(\theta^{\prime}, \theta\right)=A_{2}\left(\theta^{\prime}, \theta\right)$ for all $\theta^{\prime}, \theta \in S^{2}$ then $q_{1}=q_{2}$. Here $A_{j}\left(\theta^{\prime}, \theta\right)$ is the scattering amplitude corresponding to $q_{j}, j=1,2$, at a fixed $\kappa>0$.

Assume that (4) holds. Our basic idea is to show that (4) implies

$$
A_{q}\left(\theta^{\prime}, \theta\right)=A_{q \circ R}\left(\theta^{\prime}, \theta\right) \quad \forall \theta^{\prime}, \theta \in S^{2} \text { and } \forall R \in \mathrm{O}(3)
$$

where $\mathrm{O}(3)$ is the group of rotations in $\mathbb{R}^{3}$. Here $A_{q}\left(\theta^{\prime}, \theta\right)$ is the scattering amplitude corresponding to the potential $q(x)$, and $q \circ R$ is the potential whose value at a point $x$ is $(q \circ R)(x):=q\left(R^{-1} x\right)$. Since $q\left(R^{-1} x\right) \in Q_{a}$ if $q(x) \in Q_{a}$, it follows from theorem 2 and equation (5) that

$$
q(x)=q\left(R^{-1} x\right) \quad \forall R \in \mathrm{O}(3) .
$$

This means that $q(x)=q(|x|)=q(r)$. Thus the proof is complete as long as (5) is established. Equation (5) follows from the equations

and

$$
A_{q \circ R}\left(R \theta^{\prime}, R \theta\right)=A_{q}\left(\theta^{\prime}, \theta\right)
$$

$$
A_{q}\left(\theta^{\prime}, \theta\right)=A_{q}\left(R \theta^{\prime}, R \theta\right) \text {. }
$$

Equation (7) holds for any $q(x)$ and is an immediate consequence of the definition of the scattering amplitude $A_{q}\left(\theta^{\prime}, \theta\right)$. Equation (8) follows from our basic assumption (4), since $\theta^{\prime} \cdot \theta=R \theta^{\prime} \cdot R \theta$ for any $R \in \mathrm{O}(3)$.

Let us explain (7) in detail. Let $A_{q}\left(\theta^{\prime}, \theta\right)$ be the scattering amplitude in a certain coordinate system, say $\tau$. Consider the same scattering amplitude in a coordinate system $\tau^{\prime}$ in which any vector which in the coordinate system $\tau$ was $x$ is equal to $R x$, where $R \in \mathrm{O}(3)$. In the coordinate system $\tau^{\prime}$ the potential $q$ evaluated at a point $y$ has the value $(q \circ R)(y)=q\left(R^{-1} y\right)$. For example, suppose that the potential $q$ evaluated at a point $x$ in the coordinate system $\tau$ has the value $q(x)=d \cdot x$, where $d$ is a constant vector and the dot denotes inner product. Then, in the coordinate system $\tau^{\prime}$, vector $d$ is equal to $R d$, so that the potential $q$ evaluated at a vector $y$ in the coordinate system $\tau^{\prime}$ is equal to $R d \cdot y=d \cdot R^{-1} y=q\left(R^{-1} y\right):=(q \circ R)(y)$. Here we use the fact that $R^{\prime}=R^{-1}$, where $R^{\prime}$ is the transposed transformation; that is, the fact that rotations in $\mathbb{R}^{3}$ are orthogonal transformations. Thus, in the coordinate system $\tau^{\prime}$ the value of the scattering amplitude $A_{q}\left(\theta^{\prime}, \theta\right)$ is equal to $A_{q \circ R}\left(R \theta^{\prime}, R \theta\right)$. This is the desired equation (7). Theorem 1 is proved.

Remark 1. Our argument shows that, for any class of potentials $q(x)$ for which the uniqueness theorem for the inverse scattering problem holds for certain data, condition (4) is necessary and sufficient for $q(x)$ to be spherically symmetric if this condition holds for the above data. For example, if (4) holds for all sufficiently large $\kappa>0$ then $q(x)=q(|x|)$ if $q$ belongs to the class of potentials for which the uniqueness of the solution of the inverse scattering problem is proved for the data $A\left(\theta^{\prime}, \theta, \kappa\right)$ given for all $\theta^{\prime}, \theta \in S^{2}$ and all $\kappa$ sufficiently large. These are potentials $q(x)$ for which $|q(x)| \leqslant c(1+|x|)^{-b}, b>3, c=$ constant $>0$; see [6] (for an even larger class of potentials with $b>1$, see [9]).

The argument in this paper is applicable to the scattering by obstacles such as, for example, in the problem considered in $[1,7,10]$. 
AGR thanks NSF, ONR and SFB 256 and OLW thanks the Fulbright Commission for support.

\section{References}

[1] Ramm A G 1989 Necessary and sufficient condition for a scattering amplitude to correspond to a spherically symmetric scatterer $A p p l$. Math. Lett. 2

[2] Ramm A G 1988 Multidimensional inverse problems and completeness of the products of solutions to PDE J. Math. Anal. Appl. 134 211-53

[3] Ramm A G 1988 Uniqueness theorems for multidimensional inverse problems with unbounded coefficients J. Math. Anal. Appl. 136 568-74

[4] Ramm A G 1988 Recovery of potential from the fixed-energy scattering data Inverse Problems 4 877-86

[5] Ramm A G 1986 Scattering by Obstacles (Dordrecht: Reidel)

[6] Ramm A G 1987 Characterization of the scattering data in multidimensional inverse scattering problem Inverse problems: An Interdisciplinary Study ed P C Sabatier (New York: Academic) pp 153-67

[7] Ramm A G 1989 Completeness of the products of solutions to PDE and inverse problems Int. Conf. on Inverse Problems (Bulgaria, September 1989), Preprint SFB 256, Bonn

[8] Ramm A G 1989 Multidimensional inverse scattering problems and completeness of the products of solutions to homogeneous PDE Z. Angew. Math. Mech. 69 T13-22

[9] Saito Y 1986 Asymptotic and approximate formulas in the inverse scattering problem for the Schrödinger operator Lecture Notes in Mathematics 1218 (Berlin: Springer) pp 190-200

[10] Ramm A G 1989 Symmetry properties for scattering amplitudes and applications to inverse problems Preprint SFB 256, Bonn 